\title{
Influence of external magnetic field on thermophysical parameters of magnetic fluid based on aqueous hydrogen peroxide or ethylene glycol with a mixture of lanthanum manganite powder and toner printer cartridge
}

\author{
Jamshed Zaripov $^{1, \mathrm{a}}$ and Boris Borisov ${ }^{2}$ \\ ${ }^{1}$ Tajik Technical University, 734042 Dushanbe, Tajikistan \\ ${ }^{2}$ Tomsk Polytechnic University, 634050 Tomsk, Russia
}

\begin{abstract}
Heat transfer agent magnetic fluids based on aqueous solutions of hydrogen peroxide or a mixture of ethylene glycol with the powders of lanthanum manganite and the toner cartridge are considered. Experimental data on the effect produced by an external magnetic field on the characteristics of magnetic fluids and heat exchanger efficiency were analyzed. As the heat exchanger is considered flat-plate solar collector. The conclusion about the possibility of using the above technologies to improve the efficiency of heat exchangers.
\end{abstract}

Efficiency of heat exchangers is largely determined by the efficiency of heat transfer processes, which in turn is closely related to the thermophysical parameters used coolants. In recent years, increasing attention to the use of magnetic fluids - liquids, fine powders which are placed, having magnetic properties. These fluids besides higher values of thermal parameters, in particular the thermal conductivity, allowing increasing the intensity of the heat flow in the heat transfer process, have the property to change their characteristics under a magnetic field. This makes it possible to regulate the heat exchange processes, which is so important for a variety of technical solutions. For use as a coolant in a variety of heat exchangers (such as solar collectors) have recently often include aqueous solutions of hydrogen peroxide or ethylene glycol. Intensify the processes of heat exchange is offered with the help of magnetic solutions added to a mixture of fine powders of toner for printer with lanthanum manganite $\left.\left(\mathrm{La}_{\mathrm{y}} \mathrm{SrMnO}_{3-\mathrm{x}}\right)(\mathrm{y}<\mathrm{x})\right)$. The ultimate goal of the research was to determine the dependence of the efficiency of the heat exchanger on the example of the solar collector on the quantitative composition of the working bodies selected as coolants and external magnetic field. Analysis of efficiency of heat exchangers performed numerically based on a mathematical model, initial parameters which were coolants thermal parameters and their changes under the influence of an external magnetic field. Specific values of the thermal parameters (in particular, the thermal conductivity, specific heat and thermal conductivity) for specific temperature conditions and the characteristics of the external magnetic field have been obtained experimentally. Specific heat $(c)$ was determined by heating a monotone and was considered to be independent of the magnetic field. Coefficient of thermal conductivity $(\lambda)$ was

\footnotetext{
${ }^{a}$ Corresponding author: bvborisov@tpu.ru
}

This is an Open Access article distributed under the terms of the Creative Commons Attribution License 4.0, which permits unrestricted use, distribution, and reproduction in any medium, provided the original work is properly cited. 


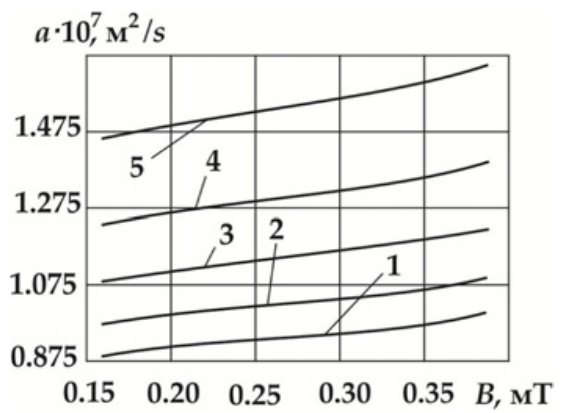

Figure 1. Dependence of the thermal diffusivity and the coolant from the vector magnetic induction B and the mass fraction of magnetic powder $\mathrm{g}$ under normal physical conditions. $1 .-\mathrm{g}=0.1 \% ; 2 .-\mathrm{g}=0.15 \% ; 3 .-\mathrm{g}=0.2 \%$; 4. $-\mathrm{g}=0.25 \% ; 5 .-\mathrm{g}=0.3 \%$.

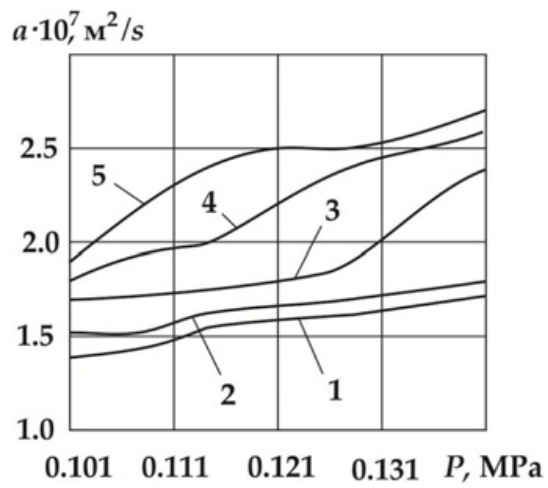

Figure 2. The dependence of the thermal diffusivity and the coolant pressure $\mathrm{P}$ and the mass fraction of hydrogen peroxide in a solution $\mathrm{n}$ at a temperature $20^{\circ} \mathrm{C} .1 .-\mathrm{n}=3 \% ; 2 .-\mathrm{n}=17 \% ; 3 .-\mathrm{n}=27 \% ; 4 .-\mathrm{n}=37 \%$; 5. $-\mathrm{n}=42 \%$.

determined by using flash upgraded laboratory equipment to explore the impact on the value of $\lambda$ as changes in coolant pressure $\mathrm{p}$ (in the range of $0.101 \div 0.141 \mathrm{MPa}$ ) and the induction vector of the external magnetic field B (in the range of $1.594 \div 3.866 \mathrm{mT}$ ). For certain values of the experiments with $\lambda$ and $c$ and thermal diffusivity $a=\lambda /(c \cdot \rho)$ values were determined for selected ranges of the external parameters.

Figures 1 and 2, shows typical results of the calculation of the thermal diffusivity of coolant from the experimentally obtained values of the thermal conductivity and specific heat. The experiments used an aqueous solution of hydrogen peroxide. Ranged percentage of hydrogen peroxide and a magnetic powder in a changing magnetic field parameters of the external pressure and in solution. For ease of analysis of experimental results in Fig. 1 shows the dependence for $a$ given pressure constancy $p=0.101 \mathrm{MPa}$ and the initial concentration of hydrogen peroxide in the initial solution $(\mathrm{n}=3 \%)$, depending on the external magnetic field and the mass fraction of the magnetic powder ( $g$ ), added to the initial solution. Figure 2 shows the results of the same series of experiments, but selected and processed for constant values of the vector magnetic field induction $B=15 \cdot 10^{-5} \mathrm{~T}$ and the mass fraction of magnetic powder $g=0.3 \%$, added to the initial solution by varying the initial mass concentration of hydrogen peroxide (up to adding magnetic powder), and pressure in the solution. Provided in the graphs curves correspond to the average of the experimental data. The error in determining $c$ and $\lambda$ no more than $5 \%$ at $95 \%$ confidence level. 


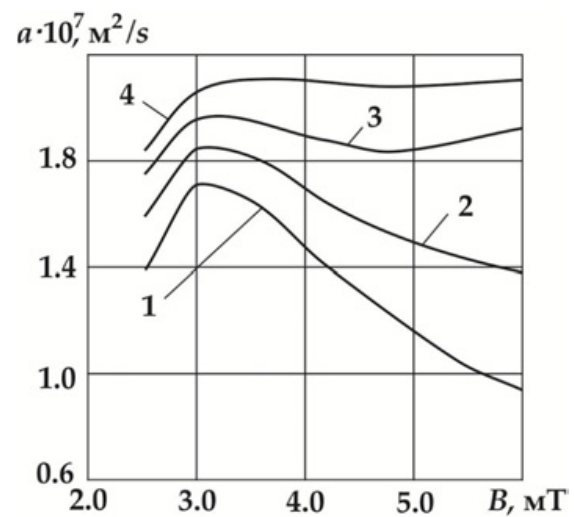

Figure 3. Dependence of the thermal diffusivity and the coolant from the vector magnetic induction $\mathrm{B}$ and the pressure $\mathrm{p}$ at a mass fraction of magnetic powder $\mathrm{g}=0.2 \%$ and temperature $20^{\circ} \mathrm{C} \cdot 1 .-\mathrm{p}=0.101 \mathrm{MPa}$; 2. $-\mathrm{p}=0.121 \mathrm{MPa} ; 3 .-\mathrm{p}=0.135 \mathrm{MPa} ; 4 .-\mathrm{p}=0.141 \mathrm{MPa}$.

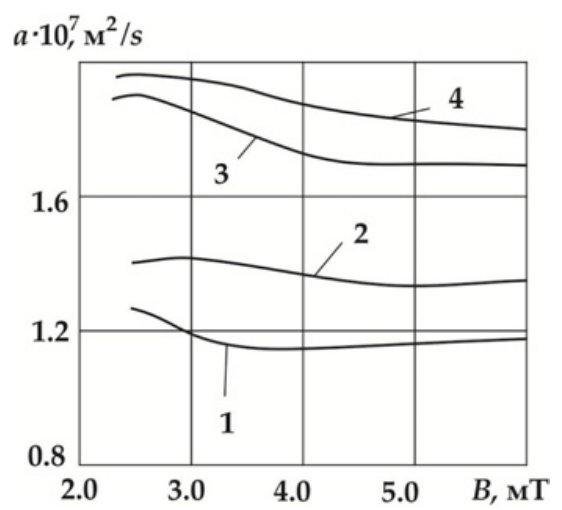

Figure 4. Dependence of the thermal diffusivity and the coolant from the vector magnetic induction $\mathrm{B}$ and the pressure $\mathrm{p}$ at a mass fraction of magnetic powder $\mathrm{g}=0.8 \%$ and temperature $20^{\circ} \mathrm{C} \cdot 1 .-\mathrm{p}=0.101 \mathrm{MPa}$; 2. $-\mathrm{p}=0.121 \mathrm{MPa} ; 3 .-\mathrm{p}=0.135 \mathrm{MPa} ; 4 .-\mathrm{p}=0.141 \mathrm{MPa}$.

The analysis of the experimental dependences shows that growth of the values of mass fractions of hydrogen peroxide, magnetic powder, magnetic induction and the pressure in the solution values in the ranges of values leads to an increase of the thermal diffusivity. The dependence of the thermal diffusivity of the magnetic field is almost linear. Increase in the magnetic field leads to the structuring of the liquid along the magnetic field lines using magnetic powder supplements. And the growth of the mass fraction of powder is understandable reinforces this trend. Analysis of the dependence of the thermal diffusivity of the pressure is more complicated. Even with the same minor changes in the level of pressure, apparently, there are complex processes in the internal structure of the solution, which is reflected in the nonlinear dependence of the specific heat, volume, and thus the density of the mass fractions of hydrogen peroxide and water.

Regularities described are relatively low values of the magnetic induction. An increase in the dependence of the thermal diffusivity for the saturation is reached with a local maximum. More explicitly, it is observed in experiments, experiments with an aqueous solution of different concentrations of ethylene glycol as a base component, and added that the magnetic powder (Figs. 3, 4). 


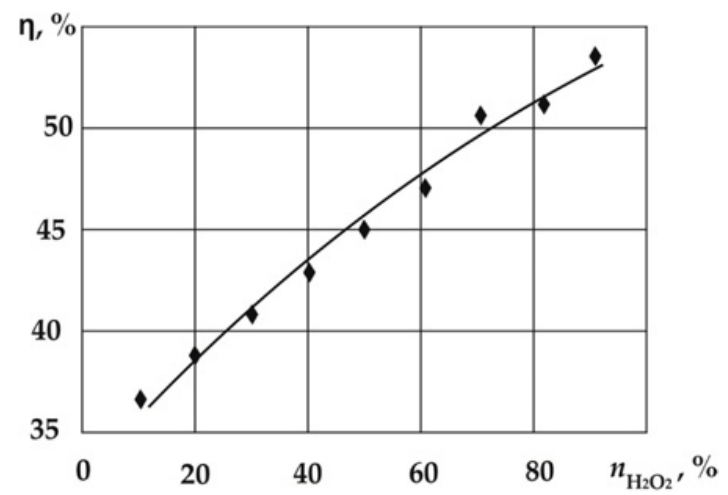

Figure 5. Dependence of the efficiency of the solar collector on the concentration of hydrogen peroxide.

According to the analysis of experimental results presented in Figs. 3 and 4, with a lower value of the mass fraction of magnetic powder in solution and a lower pressure with increasing magnetic induction after reaching a maximum value of the thermal diffusivity and located in area $\mathrm{B} \approx 3 \mathrm{mT}$, there comes a significant portion of lower-value and. With increasing pressure oscillation in the solution values and expressed not so explicitly. With the trend to achieve a certain constant value. Increasing the mass fraction of magnetic powder $g$ leads to some shift of the maximum value sof the thermal diffusivity and that can be achieved at lower values of the magnetic field induction. However, the peak in the curves and $(B)$ with increasing values of $g$ less explicitly expressed.

It should be noted that the value of mass fraction $g$ is negligible and does not affect the flow hydrodynamics. That is, the hydraulic path loss in the heat exchanger with the addition of the coolant of the magnetic powder increases slightly.

The obtained parameters $(c, \lambda$ and $a$ ) were used in the numerical bypass solar collector. Authors used the program, the results of which were tested by calculations on the European software product «SUNMEDIA». Calculations have shown similar results. The numerical analyzes influence of the concentration of hydrogen peroxide on the efficiency $\eta$ of the solar collector shown in Fig. 5. For ease of analysis, these calculations correspond to constant values of $g=0,2 \%$ and $\mathrm{B}=3 \mathrm{mT}$. According to the research, the following conclusions:

1. The use as coolants instead of water of aqueous solutions of hydrogen peroxide and ethylene glycol increases the efficiency of heat exchangers;

2. Adding to the above-described aqueous solutions of the magnetic powder and at the same time using the external magnetic field can cause a substantial change in thermal and thermodynamic parameters of the heat transfer fluids. These changes, in general, non-linear, which makes it possible to find their optimal values for improving the efficiency of heat transfer data.

\section{References}

[1] B. M. Berkovskii, V. F. Medvedev, N. S. Creeps, Magnetic fluids. Moscow, (1989)

[2] Y. I. Dikanskii, Experimental study of magnetic fields in the liquid. Magnetohydrodynamics 3, 4 (1982) 